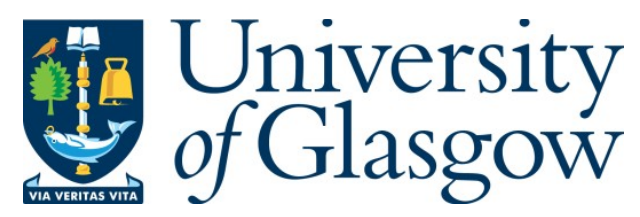

Flores Saldivar, A., Goh, C. S., Li, Y., Chen, Y., and Yu, H. (2016) Identifying smart design attributes for Industry 4.0 customization using a clustering Genetic Algorithm. In: 22nd International Conference on Automation and Computing (ICAC), 2016, University of Essex, Colchester, uk, 7-8 Sept 2016, pp. 408-414. ISBN 9781862181328.

There may be differences between this version and the published version. You are advised to consult the publisher's version if you wish to cite from it.

http://eprints.gla.ac.uk/130848/

Deposited on: 25 November 2016

Enlighten - Research publications by members of the University of Glasgow http://eprints.gla.ac.uk 


\title{
Identifying Smart Design Attributes for Industry 4.0 Customization Using a Clustering Genetic Algorithm
}

\author{
Alfredo Alan Flores Saldivar ${ }^{1}$, Cindy $\mathrm{Goh}^{1}, \mathrm{Yun} \mathrm{Li}^{1}, \mathrm{Yi} \mathrm{Chen}^{2}$, and Hongnian $\mathrm{Yu}^{3}$ \\ ${ }^{1}$ School of Engineering, University of Glasgow, Glasgow G12 8LT, U.K. \\ ${ }^{2}$ College of Computer Science and Technology, Dongguan University of Technology, Dongguan, Guangzhou 523808, China \\ ${ }^{3}$ Faculty of Sciences \& Technology, Bournemouth University, Talbot Campus, Poole BH12 5BB, U.K. \\ Email: a.flores-saldivar.1@ research.gla.ac.uk; Yun.Li@glasgow.ac.uk (corresponding author)
}

\begin{abstract}
Industry 4.0 aims at achieving mass customization at a mass production cost. A key component to realizing this is accurate prediction of customer needs and wants, which is however a challenging issue due to the lack of smart analytics tools. This paper investigates this issue in depth and then develops a predictive analytic framework for integrating cloud computing, big data analysis, business informatics, communication technologies, and digital industrial production systems. Computational intelligence in the form of a cluster k-means approach is used to manage relevant big data for feeding potential customer needs and wants to smart designs for targeted productivity and customized mass production. The identification of patterns from big data is achieved with cluster k-means and with the selection of optimal attributes using genetic algorithms. A car customization case study shows how it may be applied and where to assign new clusters with growing knowledge of customer needs and wants. This approach offer a number of features suitable to smart design in realizing Industry 4.0.
\end{abstract}

Keywords- Smart manufacturing, Industry 4.0, smart design, design and manufacture, big data analytics, cluster k-means, genetic algorithm.

\section{INTRODUCTION}

Mass customization, in manufacturing terms means automated tailor-made products. Explained by Kull [1], mass customization represents the combination of two concepts: smart factory and direct digital manufacturing (DDM). DDM is found in a digital form, representing the design of a product which is passed onto the suppliers. Then in an individual fashion, suppliers add specific data to produce components, and smart factories hopefully mass customize and mass produce automatically. This is visible along the horizontal integration path of the future Industry 4.0 (i4) value chain [2]. This also introduces Internet of Things (IoT) to manufacturing by connecting embedded systems via the Internet and smart production processes to pave the way for a new technological era expected to transform industry and business models radically [3].

The foundation of $i 4$ is the combination of strengths from optimized industrial manufacturing with innovative internet technologies [2], and is hence growing rapidly [4]. In i4, a collection of technologies and concepts such as Cyber-Physical Systems (CPS), Smart Factories, IoT, and Internet of Services (IoS) interact with one another to form a closed-loop production value chain. Communications between these components can take place at several levels [5]. For example, within a modular structured smart factory, CPS monitor the physical processes, create a virtual copy of the physical world and make decentralized decisions. Over the IoT, CPS communicate and cooperate with each other and with humans in real time. Via the IoS, both internal and cross-domain organizational services are offered and utilized by participants of the value chain [6].

While integration of methodologies for smart manufacturing has been spearheaded by the German industry at present, there emerges so far little industrial or academic research pursuing service-oriented strategy and customization prior to customized products can actually be manufactured. This can only be achieved with the introduction of methods that can enable adaption, self-aware, self-learning, self-optimized, selfpredictive, self-maintained and self-configurable automation technology aiming to change how services are provided and ways products are manufactured [7]. If companies acquire the power of big data, their challenge can be dealt with, thereby addressing issues like customers' potential needs and wants. The ability to predict key consumer trends with needs and wants is an advantage that would put companies not only ahead of their competitors, but also i4-ready. However, due to the lack of smart analytic tools for design and manufacture, most manufacturing systems are not ready to take advantage of the vast information for i4 as yet.

In this paper, an approach which focuses on predictive and selective tools for customer needs and wants is developed. This approach is proposed under i4 principles because of the capacity of integration with big data analytics, cloud computing, communication technologies, business informatics and CPS in industrial production systems. With value-add to the product right from the design stage, this approach addresses automation in predicting customers' needs and wants by selecting optimal product design triggering the customization of products and changing the way products are built. The aim of this paper is to predict customers' needs and wants, using cluster k-means and Genetic Algorithm (GA) selection for customized designs for smart manufacture.

Challenges addressing customer relations in i4 is presented in Section II, including mass customization issues, business intelligence and smart data. Section III presents methodology and tools used to achieve the prediction, where the framework for achieving mass customization is also presented. Application case study results are given in Section IV. Finally, Section V draws conclusions and highlights future work.

\section{CHALLENGES ADDRESSING CUSTOMER RELATIONS IN INDUSTRY 4.0}

The use of i4 principles is gaining popularity in the manufacturing sector. Nevertheless, development in this field is 
still in its infancy. To progress from the nascent phase to become a fully integrated reality will require several technologies to become sufficiently mature. This requires not only machines, but also individual parts to become connected and smart [8]. A smart factory needs to combine the use of IoT in the production cycle, and to include the supply chain on the cloud, where cooperative design and construction on the cloud takes part.

Social media networks and information technology have increasingly influenced customers' perceptions on product innovation, variety, quality and speed of delivery. With increasing customer demands, a company can adapt and respond to changes more readily. Mass customization influences the potential use of i4 in a positive way. It allows individual reactions on customer requirements with a higher degree of self-organization [5]. Because products bought online often do not meet individual preferences, customers could return those products. The next section attempts to address these main issues in achieving mass customization.

\section{A. Significant problems achieving customization}

Part of the main trends and expected developments for I4 is creating different value factors where products are considered based on the idea of batch-size-of-one manufacture according to individual requirements of the customer [2]. With the concept of mass customization of the product/service the customer is also integrated in the value chain. The physical product is also combined with new services that offers functionality and access rather than product ownership to the customer together with the new way of making business and its models [9].

In [10], the opportunities i4 has on sustainable manufacturing, both from the macro perspective and the micro perspective are summarised as follow:

- Business models: New evolving business models in i4 are highly driven by the use of smart data for new services. In the long-term, sustainable business models are necessarily characterized by competitiveness. In the same way, selling the accessibility and functionality of products instead of selling only tangible products will be a leading concept [10].

- Value creation networks: Cross-linking to create value networks in i4 gives new opportunities for realizing closed-loop product life cycles and industrial cooperation. It also allows efficient coordination of the material, product, and energy throughout the product life cycles as well as between different factories. Closed-loop life cycles also enable multiple use phases with remanufacturing or reuse in between [10].

- Products: The approach for design of products in i4 is focused on realizing closed-loop life cycles for products by enabling the re-manufacturing and re-use of specific products or by applying cradle-to-cradle principles. As the final outcome of the manufacturing process, product quality can provide much insight on machine condition via backward reasoning algorithms.

- Processes: The design of appropriate manufacturing process chains through the introduction of new data driven and cloud-enabled technologies is key for achieving selfaware and self-learning machines. The importance of leveraging on additional flexibility and capabilities offered by cloud computing is imminent, but adapting prognostics and health management algorithms to efficiently implement current data management technologies requires further research and development.

Summarising what authors in [11] and [10] address when trying to achieve mass customization, it becomes clear how data is managed represents a key role. CPS under the i4 vision will implement mass production and intercommunication through the IoT, but mass customization needs to be designed in advance. It is found that very often customers are not clear of their needs and wants [12]. Suddenly how data is managed will lead to evolution for the innovation floor by this constant communication and linkage that IoT enables, but as well data aim to move from manual innovation to the automation of this process. Moving to automated selection of patterns and attributes to design customized products is the purpose of this work.

\section{B. Smart information achieving prediction}

The use of smart data from big data analytics is critical to achieve accurate prediction. According to [13], many manufacturers have large amounts of data available, but are limited in their ability to analyse and effectively translate them into revenue. The concept of smart data enables real-time data stream analysis, providing enormous improvement in real-time problem-solving as well as cost prevention. According to this, it can be assumed that smart data and analytics will be the foundation for areas such as: proactive maintenance, forecasting and automation. In a practical case study provided in [13], a company named ConAgra Mills make 800 different kinds of flour for its clients. They introduce a predictive tool named: "building smarter manufacturing with the internet of things"; in this way customer demand, capacity requirements and pricing forecast is achieved, and the way decisions are made increased production capacity utilization in 5\% as well as maximize revenues.

Knowledge captured in data can be modelled by pattern recognition. Pattern is described here as a representation of an event in the form of data or a succession of events in the physical world. When dealing with data analytics, it is necessary to make a distinction between descriptive and predictive analytics. Descriptive analytics aims to condense data and pattern recognition, then those patterns form the foundation of predictive analytics. Sketching a number of different techniques (e.g., modelling, machine learning and statistical methods), it is possible to predict what might happen in the future based on probability of reliability. The current flow of data is then analysed to identify known patterns for prediction of future similar events [13]. Big data and cloud computing for $i 4$ are viewed as data services that utilize the data generated in i4 implementations, but are not independent as i4 components [6]. For i4 and smart manufacturing processes, dealing with large data storage, sharing data, processing and analysing are becoming key challenges to computer science research. Some examples of these include efficient data management, additional complexity arising from analysis of semi-structured or unstructured data and quick time critical processing requirements.

Different perspectives for achieving prediction using smart data are presented in [14], y targeting optimization and achieving prediction using what if scenarios. It is described that real-time business-intelligence is based on determine how customer satisfaction changes. Providing customer satisfaction, 
the problem can be seen as target location, so that objective function can be used to resolve a maximization function (profits and customer satisfaction) or minimization function (costs, waste, and non-conformities). From the pro-active perspective companies should use tools for prediction to make the right decisions at the right time. By predicting the development of external influences and propagation of predictions through the performance framework, this can be achieved. Performance of the business can be predicted by selecting the correct settings of business levers.

Transformation of the production factory into innovation floor is also proposed in [11]. In this work, it is proposed that intelligent analytics and CPS become one through the use of sensors, virtual platforms, simulation and collection of data for predict future events. A protocol presented in [15] developed by MTConnect ${ }^{\circledR}$ and Open Platform Communications (OPC) can help users record controller signals. This transforming agent consists of many components: visualization tools, predictive analytics, and an integrated platform. The development needs the use of advanced prediction tools in order to continuously process data into useful information that can explain any uncertainty in the system and thereby enable staff members to make more informed decisions.

\section{Business Intelligence for Industry 4.0}

Business intelligence refers to access, capture and analyses of information to enable precious assets of a company, i.e. from raw data to drivable information to improve business performance [16]. A typical business intelligence features include trend analysis, reporting and visualization, predictive analysis, and customer behaviour analysis as illustrated in Figure 1. Three main categories are presented: analytical tools, data warehouses and reporting tools.

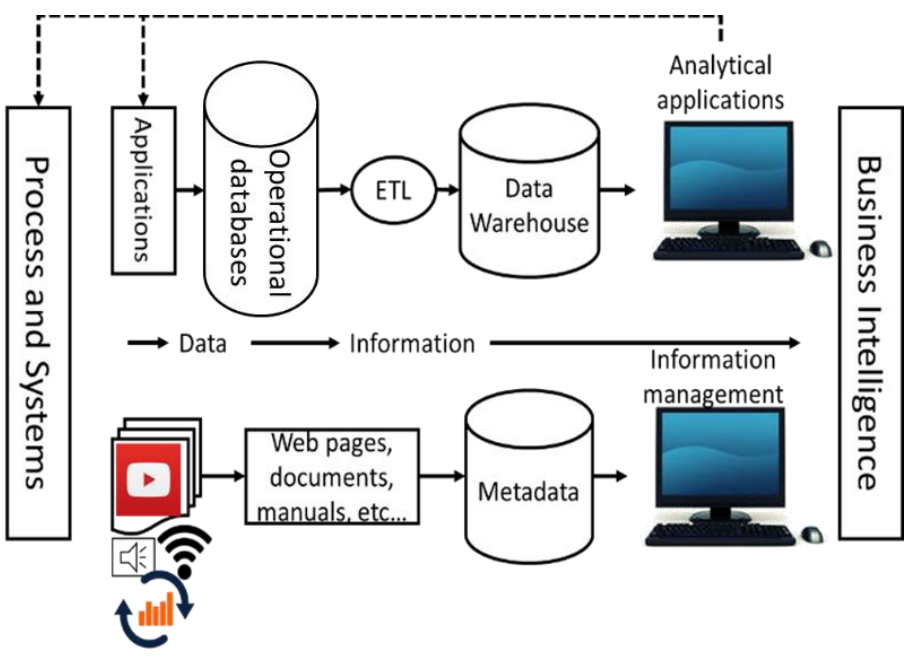

Figure 1. Business intelligence - turning data into information and action

$$
\text { [16]. }
$$

In this framework, data warehouses collect data from different sources including databases and unstructured text, and integrate them for subsequent analysis. Outputs are produced from visualization and reporting tools based on the information from customers. Those tools generate dashboards and reports with different degrees of detail and occasionally drilldown capabilities [16].

From a smart manufacturing perspective, it is discussed in [14] that customers play a key role in the process by improving customer-business relations, but also business should be responsive to take necessary actions in real-time considering the entire customer lifecycle. The implementation of business intelligence is not a trivial task that can be completed overnight. This can be attributed to two main factors:

i. The lack of an automated closed-loop feedback system that can intelligently inform business processes to respond to changes in real-time based on the inputs (for example, data trends, user experience, etc.) received.

ii. Existing analytical tools cannot accurately capture and predict consumer patterns.

The possible use of digital models is a way forward to (i) - a digital model capable of achieving an automated closed-loop. In i4, better designs are evolved from existing web-based technologies, internet services and internet market places where digital products are used as starting points. Companies will have to be prepared for a digital transformation, so they can get the best out of i4 technologies.

For (ii), a possible solution when analysing business contained in data utilizing intelligence, is to view them as the use of gathered information into data finally into action. In this way, the intelligence comes from the expert knowledge that can also be integrated in the analysis process, the knowledge-based methods used for analysis, and the new knowledge created and communicated by the analysis process.

Moving forward with i4 principles in business intelligence approaches, [14] presents the i4 innovation cycle for developing existing business and new services. It comprises three phases that companies experiment in one continuous process for innovative design as shown in Figure 2.

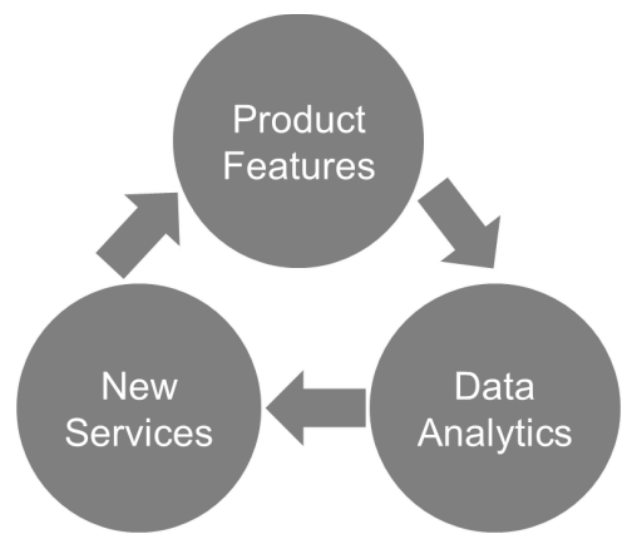

Figure 2. Industry 4.0 innovation cycle [13].

In the product features phase, the purpose is to equip the components and machine with i4 features that include actuators, sensors and information processing system, as well as customized application software. In addition to this, the components and machines require a network interface to provide them with wired or wireless network connection in the area of the application so that they can be accessed.

\section{Methodology AND TOOLS}

In this work, concepts like data analytics, customer needs and wants, and prediction are integrated into one framework under the principles of smart manufacturing and i4 Many researchers have concluded that automation together with a predictive closed-loop is ideal for customized designs. In this section, the approach developed and the analytic tools used to achieve customized designs are described.

\section{A. Simple k-means approach}

$\mathrm{K}$-means clustering is a partitioning-based clustering technique, it classifies/groups items into k-groups (where $\mathrm{k}$ is 
specified number of clusters) [17]. In this work, the WEKA toolbox in Matlab [17] is used: 1) Arbitrarily choose k objects from $\mathrm{D}$ as the initial cluster centres, 2)Repeat, 3)Assign/reassign each object to the cluster to which the object is most similar, based on the mean value of the objects in the cluster, 4)Update the cluster means i.e. calculate the mean value of the objects for each cluster, and 5) Repeat until no change.

This method is characterized by the following features:

- The algorithm attempts to determine $K$ partitions, minimizing the squared error functions.

- It is efficient and relatively scalable when processing large data sets because of the complexity of the algorithm is $O(n k t)$ where $N=$ total number of objects; $K=$ number of clusters; and $T=$ number of iterations.

- This method frequently terminates at the local optimum.

It is known that centroid based technique which takes the $k$ parameter, and partitions a set of $n$ objects into $k$ clusters so that the resulting intra-cluster similarity is low. Clustering similarity is measured taking the mean value of the objects in cluster which can be seen as cluster's centroid or gravitational centre.

K-means clustering works as well with Euclidean distance. In this case, the distance between two points in the plane with $(x, y)$ and $(a, b)$ coordinates is given by:

$$
\operatorname{dist}((x, y),(a, b))=\sqrt{(x-a)^{2}+(y-b) 1^{2}}
$$

\section{B. Tools for select best attributes}

Genetic Algorithms (GA), a method which simulates the processes in natural evolution based on the Darwinian principle of "survival of the fittest" [18] is well known for its global search capabilities. The GA is used in this work to search for the best attributes.

GA is primarily composed of three operators: crossover, mutation, and reproduction. Crossover combines good chromosomes or strings to try to generate better offspring's; reproduction selects best subset of input attributes (string); mutation alters a string locally to attempt to create a better string. The string or chromosome consists of binary bits: 1 to represent selection of attribute else 0 to drop that attribute. In each generation, the population is evaluated and tested until the algorithm terminates. If the termination or target criterion is not satisfied, the population is operated upon by the three GA operators and then re-evaluated. This process is repeated for specified number of generation. How GA was used for selecting the best attributes for the purposes of this work is described in Figure 3.

\section{Framework for predicting potential customer needs and wants}

Figure 4. presents the framework that can help to solve several of the afore-mentioned challenges in i4. This is based on i4 and Smart Manufacturing key objective, i.e. achieve selfprediction, and self-configurable in order to manufacture products and provide services that highly bespoke at mass production rates.

The users' inputs and requirements are key to any successful design. In the first block of the proposed framework, customer needs and wants are first captured and processed to extract key design characteristics. These information are then fed into a Computer Automated Design (CAutoD) engine [19] where the design requirements, features and performance objectives are mapped into 'genotypes' for further analyses. This process, which is commonly known as rapid virtual prototyping uses intelligent search algorithms such as the GA to explore the design search space for optimal solutions. In the proposed framework, this process takes place over the Cloud and produces a set of optimized virtual prototype at the end of the search.

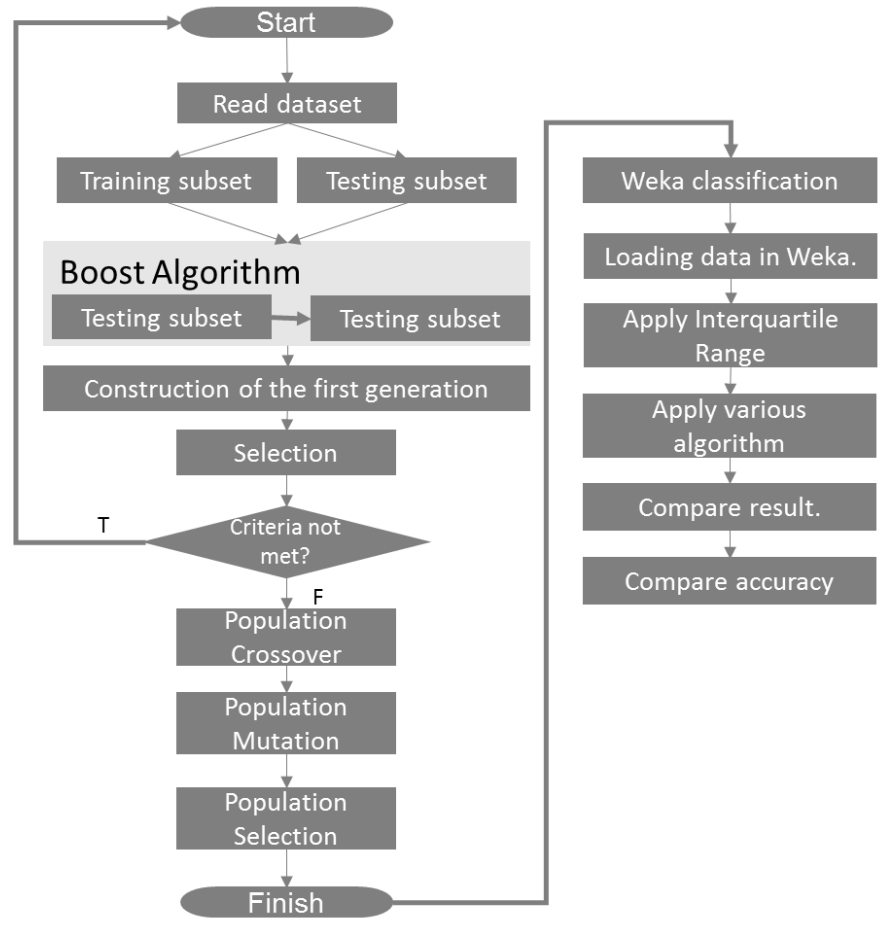

Figure 3. WEKA GA selection framework [18].

The second block of the closed loop in Figure 4. shows the virtual prototype, which is obtained from the selection and design process in CAutoD Through the integration of CPS or Cyber-Physical Integration (CPI), the virtual prototype in the second block is transformed into a physical product, i.e. the Smart Product as shown in Figure 4.

The next part of the framework refers to Business Informatics and how the Smart Product is connected to the IoT. Here is where big data takes part, through the performance of the product and the feedback from the customer, more features can be considered, this covers the necessary attributes that makes the product be manufactured in optimal ways.

Following this, the response obtained from the customer is automatically fed back to the system for further analysis and to fine-tune the virtual prototype. It is necessary to perform analysis. This analysis is related to prediction, by using node or dynamic analysis that can perform clustering, selection and detection of patterns and visualize it. After that, the SOM clustering completes update of selected attributes by comparing the latest input to the existing cluster and tries to identify one cluster that is most similar to the input sample using multidimensional distance measurement. Then several features are fed back into the cloud again.

The analysis can result in two outcomes: (i) Similar clusters found. If it is the case, this will be reflected as an existent attribute and the algorithm will update the existing cluster using information from the latest sample. (ii) Non-similar clusters found. The algorithm will hold its operation with the current sample until it sees enough count-of-cluster samples. 
When the number of out-of-cluster samples exceeds a certain amount, it means that there exists a new behaviour in the data that has not been modelled, then the algorithm will create a new cluster to represent such new behaviour. Solving a clustering problem with a cluster k-means accessed in Weka toolbox for Matlab® platform was used for training the data that is presented in the following section. For those cases, cluster k-means can be very adaptive to new conditions. Selfgrow clusters will be used as the knowledge base for customization assessment.

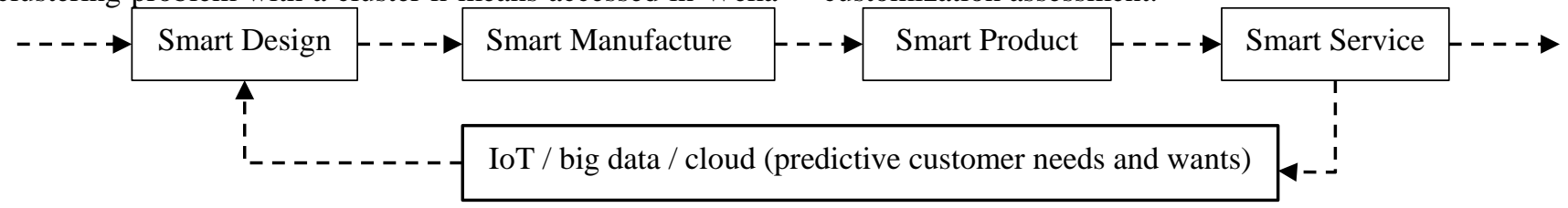

Figure 4. Industry 4.0 value chain with predictive customer needs and wants fed back for automated customization

\section{Application CASE StUdy Results}

It has been accessed to trained data found in a machinelearning repository [20] in order to run some tests, the information of the data shows an evaluation model of cars by acceptability, overall price, buying price, price of maintenance, technical characteristics, comfort, number of doors, persons capacity to carry, and safety of the car. The data set comprises of 1728 inputs and each record contains the subsequent attributes: safety, capability describing the persons to hold, buying price, maintenance price, number of doors, the dimensions of baggage boot, and car acceptance. For this data set, the attribute of car acceptance is a category label used to classify the level of the car that customers accept, then different attributes are seen as predictive inputs. The car evaluation data contents are shown in TABLE I.

TABLE I. CAR EVALUATION DATA

\begin{tabular}{|l|l|l|}
\hline $\begin{array}{l}\text { Attribute } \\
\text { name }\end{array}$ & Description & Domain \\
\hline safety & $\begin{array}{l}\text { Safety } \\
\text { evaluation }\end{array}$ & Low / med / high \\
\hline person & $\begin{array}{l}\text { The number of } \\
\text { passenger }\end{array}$ & 2 / 4 / more \\
\hline b_price & Buy Price & v-high / high / med / low \\
\hline m_price & Repair price & v-high / high / med / low \\
\hline size & $\begin{array}{l}\text { Suitcase } \\
\text { capacity }\end{array}$ & Small / med / big \\
\hline door & $\begin{array}{l}\text { The number of } \\
\text { the door }\end{array}$ & 2/3 / 4 / 5-more \\
\hline class & $\begin{array}{l}\text { level of } \\
\text { customer } \\
\text { acceptance }\end{array}$ & $\begin{array}{l}\text { Unacc / acc / good / } \\
\text { vgood }\end{array}$ \\
\hline
\end{tabular}

In order to examine the distributions for knowing this dataset better in Figure 5. presents the distributions for the car evaluation set. As a methodology to follow it was also considered the business problem as the following question: what reasonably cars can get good assessment? This question then these evaluations are used as target attributes, depending on which attribute. Then as the data mining problem, which is: find out the rules form other attributes.

\section{A. Attributes clustering using k-means approach}

Clustering was obtained using the WEKA toolbox in Matlab, in this case it was tested against the simple k-means scheme for better results. To assess the model, TABLE II. shows the results using simple K-means. Two clusters were selected and
10 seeds, and it was initially ran with the training set, where all 7 attributes and 1728 instances were considered. Results are presented in TABLE II.

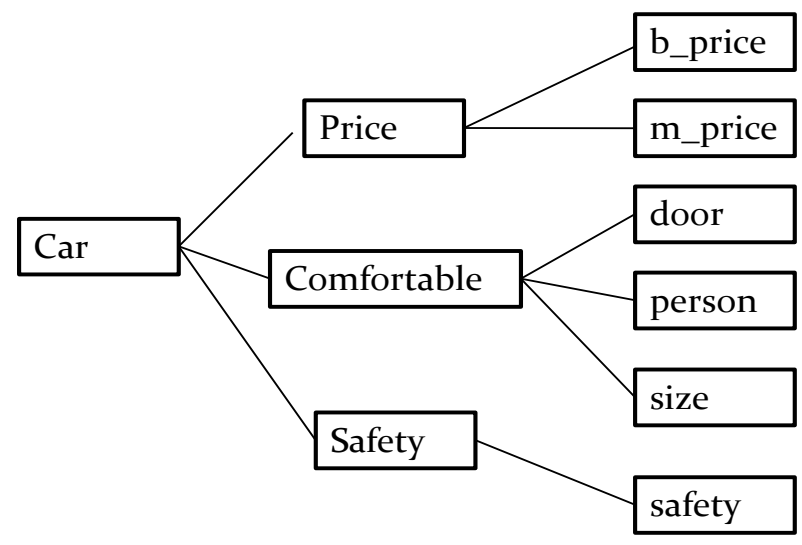

Figure 5. Distributions of car evaluation dataset for customization

TABLE II. SIMPLE K-MEANS CLUSTERING TESTING 7 ATTRIBUTES

\begin{tabular}{|c|c|c|}
\hline Instances & Percentage & Classified \\
\hline 1104 & $64 \%$ & Cluster 0 \\
\hline 624 & $36 \%$ & Cluster 1 \\
\hline
\end{tabular}

Following this, classification of clusters, as the selected mining method to build the model was also performed. Classifier decision tree (ID3) was selected, because it showed better results. The results of the model accuracy by class attribute are presented in TABLE III.

\begin{tabular}{|c|c|c|c|c|c|}
\multicolumn{5}{c|}{ TABLE III. } \\
\begin{tabular}{|c|c|c|c|} 
TP \\
Rate
\end{tabular} & $\begin{array}{l}\text { FP } \\
\text { Rate }\end{array}$ & $\begin{array}{l}\text { Preci } \\
\text {-sion }\end{array}$ & Recall & $\begin{array}{l}\text { F- } \\
\text { Measure }\end{array}$ & Classified \\
\hline $\mathbf{1}$ & 0 & 1 & 1 & 1 & unacc \\
\hline $\mathbf{1}$ & 0 & 1 & 1 & 1 & acc \\
\hline $\mathbf{1}$ & 0 & 1 & 1 & 1 & good \\
\hline $\mathbf{1}$ & 0 & 1 & 1 & 1 & vgood \\
\hline
\end{tabular}

In TABLE IV. the confusion matrix for this classification using the ID3 method in Weka toolbox is presented.

TABLE IV. CONFUSION MATRIX FOR CLASSIFIED ATTRIBUTES.

\begin{tabular}{|l|l|l|l|l|}
\hline $\mathbf{a}$ & $\mathbf{b}$ & $\mathbf{c}$ & $\mathbf{d}$ & Classified \\
\hline $\mathbf{1 2 1 0}$ & 0 & 0 & 0 & $\mathrm{a}=$ unacc \\
\hline $\mathbf{0}$ & 384 & 0 & 0 & $\mathrm{~b}=$ acc \\
\hline $\mathbf{0}$ & 0 & 69 & 0 & $\mathrm{c}=\operatorname{good}$ \\
\hline $\mathbf{0}$ & 0 & 0 & 65 & $\mathrm{~d}=\operatorname{vgood}$ \\
\hline
\end{tabular}

For this classification there were no incorrect classified instances, and when ran in Matlab the time to build the model 
was 0.01 seconds. It can be inferred form TABLE III. TABLE IV. that the classification model works well - instances assigned to domain unacceptable (unacc) turns to be the ones that have more impact on cluster assignation with a value of 1210 instances. Simple k-means clustering and ID3 show that classification of domains for each attribute can reflect the exact quantity of clusters. On the other hand, attributes like maintenance, buying and doors show more incorrect clustered instances.

According to [17] $k$-means clustering can represent weaknesses: a)With fewer samples of data, initial grouping will determine the cluster significantly; b)The number of clusters, $\mathrm{k}$, must be determined beforehand; c)With fewer samples of data, inaccurate clustering can occur; d) It cannot be inferred which variable contributes more to the clustering process since it is assumed that each has the same weight; e) The accuracy of mathematical averaging weakens because of outliers, which may pull the centroid away from its true position; and f) The results are clusters with circular or spherical shapes because of the use of distance.

\section{B. Attributes selection using a genetic algorithm}

Once clusters where found the following step was to use Coefficients Subset Evaluation (CfsSubsetEval) that according to [17] and libraries inside WEKA toolbox, means that: evaluates the worth of a subset of attributes by considering the individual predictive ability of each feature along with the degree of redundancy between them. And as a search method it was used Genetic Search, with a probability of search equals to 0.6 , a maximum of generations of 20 , mutation probability of 0.033 , population size of 20 , report frequency of 20 , number of seeds equals to 1 and starting set number 1 . For the mentioned criteria, it was disregarded the attribute class. The results obtained show that safety was the best attribute. Different to what it was obtained for class attribute, disregarding safety as the main attribute, it was selected with a higher level of prediction the class attribute. Disregarding all the remaining attributes (buying, paint, doors, persons, and lug_boot) present the same selection: class. Based on the results presented above, it is clear that the best selected attribute is class.

\section{CONCLUSION AND FUtURE DiRECTIONS}

Achieving mass customization and predicting customers' needs and wants are main issues to tackle in addressing smart manufacturing and i4. In this work, an integrated platform is developed. An application is tested in a case study, which enables pattern recognition by utilizing cluster k-means and a selective genetic algorithm.

Through the development of predictive tools for subconscious customers' needs and wants, selection of best design can be achieved in a smart way. The following features are encountered through the development of this work:

1) A smart product may be obtained with business informatics and reliable data constantly, which can be fed back to smart design again with IoT in the loop of the i4 value chain.

2) Cloud-enabled connection enhanced by the "Internet of Everything", making faster decisions and selections to satisfy customer needs and wants.

3) Decision for the manufacturer becomes easier to make, with customer-driven informatics, design and automation
4) The strong relation found in class attribute tells the car manufacturers how the level of customer acceptance influences the decision of buying a car.

5) Second strong relation is found in safety attribute, telling car manufacturers to double efforts on specific areas of opportunity.

6) Selection of best attributes worked good, and similar to cluster analysis, attribute selection followed same pattern.

7) Big data analytics (nodes) help visualize the influence of product characteristics, clustering and interpretation of subconscious customer needs and wants.

Future directions for a higher scalable application on predictive way to select attributes lead to focus on powerful tools like fuzzy logic for fuzzified mass customization. The MapReduce approach can also be considered when dealing with real-time businesses, because of the quick response and the inclusion of parallel computing as well.

\section{REFERENCES}

[1] Kull, H., Introduction, in Mass Customization: Opportunities, Methods, and Challenges for Manufacturers. 2015, Apress: Berkeley, CA. p. 1-6.

[2] Kagermann, H., W. Wahlster, and J. Helbig, Recommendations for implementing the strategic initiative INDUSTRIE 4.0. ACATECH NATIONAL ACADEMY OF SCIENCE AND ENGINEERING, 2013(6).

[3] MacDougall, W., INDUSTRIE 4.0 SMART MANUFACTURING FOR THE FUTURE. MECHANICAL \& ELECTRONIC TECHNOLOGIES, GERMANY TRADE \& INVEST, 2014(3): p. 40.

[4] Evans, P.C. and M. Annunziata Industrial Internet: Pushing the Boundaries of Minds and Machines. GE imagination at work, 2012. 21, 37.

[5] Schmidt, R., et al., Industry 4.0 - Potentials for Creating Smart Products: Empirical Research Results, in Business Information Systems: 18th International Conference, BIS 2015, Poznań, Poland, June 24-26, 2015, Proceedings, W. Abramowicz, Editor. 2015, Springer International Publishing: Cham. p. 16-27.

[6] Hermann, M., T. Pentek, and B. Otto, Design Principles for Industrie 4.0 Scenarios: A Literature Review. Technische Universitat Dortmund, 2015. 01(4): p. 4-16.

[7] Kagermann, H., Change Through Digitization-Value Creation in the Age of Industry 4.0, in Management of Permanent Change, H. Albach, et al., Editors. 2015, Springer Fachmedien Wiesbaden. p. 23-45.

[8] Kull, H., Intelligent Manufacturing Technologies, in Mass Customization: Opportunities, Methods, and Challenges for Manufacturers. 2015, Apress: Berkeley, CA. p. 9-20.

[9] Coetteleer, M., J. Holdowsky, and M. Mahto, Additive Manufacturing paths to performance, innovation, and growth. Deloitte Review, 2014. 1(19): p. 32.

[10] Stock, T. and G. Seliger, Opportunities of Sustainable Manufacturing in Industry 4.0. Procedia CIRP, 2016. 40: p. 536-541

[11] Lee, J., H.-A. Kao, and S. Yang, Service Innovation and Smart Analytics for Industry 4.0 and Big Data Environment. Procedia CIRP, 2014. 16(1): p. 3-8.

[12] Isaacson, W. The real leadership lessons of Steve Jobs. Harvard Business Review, 2012. 4, 92-102.

[13] Möller, D.P.F., Digital Manufacturing/Industry 4.0, in Guide to Computing Fundamentals in Cyber-Physical Systems: Concepts, Design Methods, and Applications. 2016, Springer International Publishing: Cham. p. 307-375.

[14] Nauck, D., et al., Predictive Customer Analytics and Real-Time Business Intelligence, in Service Chain Management, C. Voudouris, D. Lesaint, and G. Owusu, Editors. 2008, Springer Berlin Heidelberg. p. 205-214.

[15] Lee, B.E., et al. MTConnect-Based Kaizen for Machine Tool Processes. in ASME 2010 International Design Engineering Technical Conferences and Computers and Information in Engineering Conference. 2010. Montreal, Quebec, Canada: American Society of Mechanical Engineers.

[16] Azvine, B., et al. Real Time Business Intelligence for the Adaptive Enterprise. in E-Commerce Technology, 2006. The 8th IEEE 
International Conference on and Enterprise Computing, E-Commerce, and E-Services, The 3rd IEEE International Conference on. 2006.

[17] Sharma Ritu (Sachdeva, Alam Afshar M., and R. Anita, K-Means Clustering in Spatial Data Mining using Weka Interface. IJCA Proceedings on International Conference on Advances in Communication and Computing Technologies 2012, 2012. ICACACT(1): p. 26-30.

[18] Priyanka, K. and B. Dr.Kavita, Feature Selection Using Genetic Algorithm and Classification using Weka for Ovarian Cancer.
International Journal of Computer Science and Information Technologies, 2016. 7(1): p. 194-196.

[19] Yun Li, K.H.A., Gregory C.Y. Chong, Wenyuan Feng, Kay Chen Tan, Hiroshi Kashiwagi, CAutoCSD-Evolutionary Search and Optimisation Enabled Computer Automated Control System Design. International Journal of Automation and Computing, 2004. 1(17): p. 76-88.

[20] Lichman, M., UCI Machine Learning Repository, U.o. California, Editor. 2013, School of Information and Computer Science.: Irvine, CA University of California, School of Information and Computer Science. 